\title{
Incidence And Study of Occupational Factors Associated With Low Back Pain In Dentists In Pune Region India
}

\author{
Sayli Paldhikar ${ }^{1}$, Samprada Bhatkar ${ }^{2}$, Snehal Ghodey ${ }^{3}$ \\ 1,2,3 (Physiotherapy Department, MAEER's Physiotherapy college, India)
}

\begin{abstract}
Background: Occupational low back pain is most common symptom among dentists. Working for long hours has its hazards involving working in a standing position and prolonged stooping over a reclined or supine patient. Also, dentist must use a variety of hand tools in a delicate manner. Objectives: 1) To find out the prevalence of low back pain (LBP) among dentists 2) To study occupational factors associated with low back pain. Materials and Methods: This was a cross sectional study carried out among dentists in pune city during the February 2010 to April 2010. Total 245dentists were selected by simple random sampling. Information was gathered with predesigned and pretested questionnaire. Prevalence LBP is defined as rate of study subjects who experienced LBP in the past one week. Roland-Morris Disability Questionnaire (RMDQ) score was used to assess physical disability due to low back pain. Study variables included were physique of dentists, demographic features (age, gender and marital status), working conditions (working hours, dental chair used, posture during the procedures), health conditions, the presence of low back pain, Roland-Morris Disability Questionnaire (RMDQ) score. Results \& Conclusion: Prevalence of LBP was found to be $62.10 \%$. Among study subjects with $L B P, R M D Q$ score averaged 5.1, Sleeplessness, duration of working $>48 \mathrm{hrs} / \mathrm{wk}$, and body mass index $(B M I)=$ $25 \mathrm{~kg} / \mathrm{m} 2$.
\end{abstract}

Keywords: Low back pain, Dentists, Roland MorrisDisability Questionnaire, Dental Chair.

\section{Introduction}

Most of people do not relish their periodic visits to the dentists nor do they eagerly await their upcoming dental appointment. In these situations, sympathy usually lies fairly and squarely with the patients. What many people fail to appreciate, however, is that these situations place considerable musculoskeletal demands on these health professionals, whenever they are treating people in an attempt to improve their quality of life. Dental professionals commonly experience musculoskeletal pain during the course of their careers. While the occasional backache or neck ache is not a cause for alarm, if regularly occurring pain or discomfort is ignored, the cumulative physiological damage can lead to an injury or a career-ending disability. The musculoskeletal health of dental professionals has been the subject of numerous studies worldwide, and their focus has been on the pain experienced by the practitioner. For the patient, a dental visit typically involves sitting in a reclined or even supine position and the dentist often has to contort the body to perform the oral work. Dental profession typically involves working in a standing position and requires prolonged stooping over a reclined or even supine patient. Also, dentist must use a variety of hand tools in a delicate manner.

The kind of posture related musculoskeletal problems, reported by dentists, are comparable to those found in other professions involved in prolonged standing work in poor posture. Thus, the dental team is at high risk of neck \& back problems due to limited work area and impaired vision associated with the oral cavity. These working restrictions frequently cause a dentist to assume stressful body postures to achieve good access and visibility inside the oral cavity. Also, dental procedures are usually long and require much more concentration during work.

Prevalence of musculoskeletal Pain among dentists in a 2010 study, Pargali [1] found that 73 percent of dentists complained of back and neck pain. Even after the evolution to seated four-handed dentistry and ergonomic equipment, studies [2-6] have found back, neck, and shoulder or arm pain present in up to 81 percent of dental operators. In the practice of dentistry, stress, tension and postural practices can contribute to back \& neck problems to the dentist. Various work-related factors among dentists have been established as predisposing the disorders i.e. overstrained and awkward back postures for back pain, repetitiveness for neck and shoulder disorders, and psychosocial stressors for back, neck and shoulder complaints [7]. A slight hand neuropathy has also been reported caused by exposure to high frequency vibration tools [8].

Musculoskeletal pain, particularly back pain, has been found to be a major health problem for dental practitioners [9-13]. Several studies have reported a similar prevalence of musculoskeletal disorders (MSD) amongst dentists. In a survey of Danish dentists for example, 50\% and 65\% reported a one year prevalence of low back pain and neck/shoulder pain, respectively [5]. A study from New South Wales (NSW), Australia, found an even higher prevalence of MSD among dentists, with $82 \%$ reporting at least one musculoskeletal symptom in the past month and 64\% reporting backache during the previous month [9]. 


\section{I.I Spinal dynamics}

In standing postures, the spine has four natural curves when viewed from the side: cervical lordosis, thoracic kyphosis, lumbar lordosis and sacral kyphosis .The curves are interdependent; achange in one curve will result in a change in the curve above or below it. Since the sacral curve is composed of five fused vertebrae, its movement is extremely limited. However, the remaining curves - especially the lumbar and cervical curves are more mobile and can be influenced more easily. When the curves of the spine are present and balanced against the center of gravity, the spine is supported mostly by the bony structures of the vertebrae resting on top of one another. When these curves become either exaggerated or flattened, the spine increasingly depends on muscles, ligaments and soft tissue to maintain erect.

The most important joint in the vertebral column is inter-body joint. A well-constructed, circular cushion of specialized fibro-cartilage called 'inter-vertebral disc' is sandwiched between adjacent bodies. These Inter-vertebral discs are of tough elastic fibers on the outside and a soft gelatin like substance mostly water, inside. The discs serve as shock absorber between vertebrae. Over time, discs lose their ability to absorb water and become less flexible. Also, small stresses and strains cause tiny tears in the outer casting of the discs. These tears heal with scar tissue which is weaker and less flexible than the original tissue. The inter-vertebral disc is a marvel of nature and can tolerate large amount of compressive loads and bending and twisting stresses. During flexion, the inter-body joint carries more weight while during extension, the facet joint carries more loads. During working, dentists maintains varying positions for prolonged periods of time, one can only speculate the amount of loading/stress suffered by the lumbar spine. The majority of mechanical failures of spinal structures results from either single or recurrent misuse of the back in this mechanically disadvantageous postures.During treatment, however, dentists should strive to maintain a neutral, balanced posture. Even with the best ergonomic equipment, operators can find themselves in sustained awkward postures. These postures often consist of forward bending and repeated rotation of the head, neck and trunk to one side .Over time, the muscles responsible for rotating the body to one side can become stronger and shorter, while the opposing muscles become weaker and elongated. The stressed shortened muscles can become ischemic and painful, exerting asymmetrical forces on the spine that can cause misalignment of the spinal column and decreased range of motion in one direction over the other. To effectively prevent injuries in dentistry, prevention strategies and ergonomic techniques must address these postural and positioning difficulties, aswell as subsequent detrimental physiological changes: muscle imbalances, stiff joints, muscle necrosis and spinal disk degeneration.

\section{I.I I Causes Of Back Problems}

Back pain is one of the most common and troublesome of complaints. Its exact cause is legion and an exact diagnosis is often difficult [14]. Back problems have been cited as the second most common reason for missing work in an industrial population. It has been stated that the most common site of pain among dentists and dental auxiliaries are in the areas of cervical \& lumbar vertebrae $[9,15,16]$. The shape of vertebral column, aging, changes, weak muscles, postural practice, movements, lifting techniques and mechanical stresshave been identifies as factors that can contribute to the neck and back pain in general [12].According to Walters, 1976, stress, postural practices (bending and twisting trying to gain better access and visibility within the oral cavity) as well as prolonged working time lead to fatigue [17]. Various other investigators have pointed out the common postural faults among dentists and dental auxiliary personal, such as craning, excessive bending and twisting of neck, bending forward from the waist, elevation of the shoulders and general bending and twisting of neck \&back [18-22].Dental auxiliaries also experienced back pain associated with their work. Though, level of pain experienced by dentists is usually greater than that felt by auxiliary personal, but as the working day progresses, discomfort experienced by both the dentist \& dental auxiliaries, significantly increases in severity [23]. In 1989, Bassette [12] concluded that despite the use of improved dental equipments, operating in a seated position using the four handed dentistry technique and increasing the frequency of exercise, the incidence of back problems has not decreased over the last 15 years. He went on to state that for many dentists, backaches are likely to be related to muscular tensions and poor working postures, Bassette, as a preventive measure, recommended that dental students should be taught relaxation techniques early in their clinical training and also they should be taught correct working positions at chair side. The most common type of back pain comes from straining the muscle bands immediately surrounding the vertebral column in lumbar region. Although such strains can occur anywhere along the spine, they happen most often in the curve of lower back and next most common site is the base of neck.

\section{Materials And Methodology}

This is a cross-sectional analysis of baseline data from a prospective study on the prevalence of low back pain in dentists among Pune region. This study was carried out in Physiotherapy department of MAEER's Physiotherapy college, Talegaon Dabhade from February 2010 to April 2010.For deciding the sample size and to check the feasibility as well as to test the questionnaire pilot study was carried out in the first week of the 
February 2010 in which the prevalence of Low back pain amongst 90 study participants was found to be $60 \%$. Based on this prevalence sample size was determined which came out to be 256 . Necessary changes were made in the questionnaire after pilot study.

Data collection: Information was gathered with the help of predesigned and pretested questionnaire. Roland-Morris Disability Questionnaire (RMDQ) score [24, 25] was used to assess physical disability due to low back pain. Study variables included were physique of dentists, demographic features (age, gender and marital status working conditions (working hours, dental chair used, posture during the procedures), health conditions, and the presence of low back pain Roland-Morris Disability Questionnaire (RMDQ) score. Dentists were selected using simple random sampling with the help of random number table. A time schedule was prepared for the study participants, so that they can participate as per their convenience. Study subjects were assured regarding confidentiality and were maintained throughout the study. The objective of the study was explained to participants and informed consent was taken. Information regarding daily working hours, daily working position, dental chair information and Roland-Morris Disability Questionnaire for Low back pain was used. The RMDQ focuses on a limited range of physical functions that were chosen as functions which would be relevant to all patients with back pain [25]. The internal consistency and responsiveness of the RMDQ had been evidenced by Crohnbach's alpha for the scale that has been estimated as $0.93,0.90,0.84[26,27]$.

Statistical Analysis: For analysis descriptive statistics used were percentage, mean and standard deviation (SD). Internal comparison was done among the study participants with LBP and without LBP with bivariate analysis using crude odds ratio, 95\% confidence interval (CI), chi-square test and unpaired student t. test. Multiple logistic regressions were used to calculate adjusted odds ratio among those factors which were found significant on bivariate analysis. All the analysis was carried out using SPSS 16.0 and EPI Info 3.5.1.

\section{Results}

Low back pain was present among 159 (62.10\%) study participants. Average RMDQ score was 5.1 and 3.9 among participants with and without low back pain respectively. The response rates to various questions in RMDQ among participants with low back pain were shown in table 1. The response rate was maximum i.e. $72.3 \%$ to the question "I change position frequently to try and get my back comfortable." Followed by response of $68 \%$ respondents to "I avoid heavy jobs around the house because of my back" (Table 1).

The bivariate analysis of attributes amongst study subjects with and without LBP. Statistically significant relationship was observed between low back pain and history of LBP, diseases other than LBP, not enough time to relax at home, sleeplessness, duration of working $>48 \mathrm{hrs} / \mathrm{wk}$., mental stress due to conflicts with the management, and BMI $\geq 25$. The factors which were found significant in bivariate analysis at the $90 \%$ significance level were entered into unconditional multiple logistic regression analysis. Table 2: shows the factors which were significantly associated with LBP after the unconditional multiple logistic regression analysis.

TABLE 1: The response rates by Roland-Morris Disability Questionnaire (RMDQ) by respondents with LBP

\begin{tabular}{|c|c|c|}
\hline $\begin{array}{l}\text { SR. } \\
\text { NO }\end{array}$ & Question & $\%$ \\
\hline 1. & I stay at home most of the time because of my back & 12.1 \\
\hline 2. & I change position frequently to try and get my back comfortable & 72.3 \\
\hline 3. & I walk more slowly than usual because of my back. & 13.2 \\
\hline 4. & Because of my back I am not doing any of the jobs that I usually do around the house. & 04.7 \\
\hline 5. & Because of my back, I use a handrail to get upstairs. & 06.8 \\
\hline 6. & Because of my back, I lie down to rest more often. & 53.8 \\
\hline 7. & Because of my back, I have to hold on to something to get out of an easy chair & 07.2 \\
\hline 8. & Because of my back, I try to get other people to do things for me. & 01.1 \\
\hline 9. & I get dressed more slowly than usual because of my back. & 03.6 \\
\hline 10. & I only stand for short periods of time because of my back. & 27.4 \\
\hline 11. & Because of my back, I try not to bend or kneel down. & 33.5 \\
\hline 12. & I find it difficult to get out of a chair because of my back. & 05.7 \\
\hline 13. & My back is painful almost all the time. & 28.3 \\
\hline 14. & I find it difficult to turn over in bed because of my back. & 04.3 \\
\hline 15. & My appetite is not very good because of my back pain. & 01.2 \\
\hline 16. & I have trouble putting on my socks (or stockings) because of the pain in my back. & 15.3 \\
\hline 17. & I only walk short distances because of my back & 24.8 \\
\hline 18. & I sleep less well on my back. & 17.2 \\
\hline 19. & Because of my back pain, I get dressed with help of someone else & 0.04 \\
\hline
\end{tabular}


20. I sit down most of the time because of my back

21. I avoid heavy jobs around the house because of my back

22. Because of my back I am more irritated and bad tempered with people than usual

23. Because of my back, I go up the stairs more slowly than usual

24. I stay in bed most of the time because of my back

TABLE2: Factors which were significantly associated with LBP

\begin{tabular}{|c|c|c|c|c|c|}
\hline Factors & \multicolumn{2}{|c|}{ With LBP } & \multicolumn{2}{|c|}{ Without LBP } & P value \\
\hline & Mean & SD & Mean & SD & \\
\hline Age & 48.6 & 07.2 & 46.9 & 06.6 & 0.060 \\
\hline BMI & 25.2 & 03.7 & 23.5 & 03.2 & $<0.001 *$ \\
\hline Working Hours (hrs/wk) & 54.8 & 08.8 & 49.4 & 09.2 & $<0.001 *$ \\
\hline Length of occupation & 24.4 & 07.4 & 19.3 & 08.3 & $<0.001 *$ \\
\hline
\end{tabular}

\section{Discussion}

In the present study prevalence of LBP was taken as low back pain during the previous week as the period of one year is too long for study subject to recall it accurately. Also in RMDQ it was mentioned as the prevalence in the previous one week. Prevalence of LBP in the present study was found to be $62.10 \%$. Prevalence of LBP in the present study was found to be $62.10 \%$. this was in accordance to a study of Prevalence of musculoskeletal Pain among dentists, by Pargali [1] who found that 73 percent of dentists complained of back and neck pain done in 2010. Also findings were similar to studies done by Walters, 1976 and studies done in New Wales and Australia[9,10].

Our study shows $65 \%$ of dentists are practicing sitting dentistry, $27 \%$ dentist are following standing dentistry and only 5\% are following both standing and sitting dentistry. Most of the young dentists follow standing position as they are unaware or negligent about pain; aged and that dentists who are practicing since many years follow sitting position because. Most of them have already suffered from back ache so they prefer sitting dentistry and it is advised ergonomically. The pain can be because of prolonged static postures or PSP, repetitive movements, suboptimal lighting, poor positioning, genetic predisposition, physical conditioning and age. .Dentists frequently assume static postures, which require more than 50 percent of the body's muscles to contract to hold the body motionless while resisting gravity. The static forces resulting from these postures have been shown to be much more taxing than dynamic (moving) forces. Dentistry needs forward bending and repeated rotation of the head, neck and trunk to one side the muscles responsible for rotating the body to one side can become stronger and shorter, while the opposing muscles become weaker and elongated[10,11]. The stressed shortened muscles can become ischemic and painful, exerting asymmetrical forces on the spine that can cause misalignment of the spinal column and decreased range of motion in one direction over the other. .One study, for example, showed that for a majority of dentists, neck rotation to the right with side bending to the left is a difficult movement to perform[22]. The cycle of muscle imbalance perpetuates as tighter muscles become tighter and weaker muscles become weaker. Even when using the best working postures, dental operators \& surgeons still maintain static contractions of the trunk muscles

\section{Conclusion}

Musculoskeletal disorders are inherent in dentistry. Serious detrimental physiological changes in the body can result from these abnormal postures, including muscle imbalances, muscle necrosis, trigger points, hypo mobile joints, nerve compression, and spinal disk herniation or degeneration. These changes often result in pain, injury particularly in the back. Preventing chronic pain in dentistry may require a paradigm shift within the profession regarding clinical work habits, including proper use of ergonomic equipment, frequent short stretch breaks and regular strengthening exercise. During clinical procedures, dentists should acquire a biomechanically ideal posture in which spinal structures are stressed least and the muscular energy spent is minimum. By acquiring a bio-mechanically ideal posture, practicing aerobic and relaxation exercises, dentists and auxiliary personnel can minimize or even prevent spine problems. In this way, they can increase their working efficiency which in turn, will help them improving the quality of dental care they can render to their patients. 


\section{Bibliography}

[1] Pargali N, Jowkar N. Prevalence of Musculoskeletal Pain among Dentists in Shiraz, Southern Iran. The Int J Occupat Environ Med 2010;1(2).

[2] Shugars DA, Miller D, Williams D, Fishburne C, Strickland D. Musculoskeletal pain among general dentists. Gen Dent 1987;35:272-6.

[3] Rundcrantz B, Johnsson B, Moritz U. Cervical pain and discomfort among dentists: epidemiological, clinical and therapeutic aspects. Part 1 -a survey of pain and discomfort.

[4] Swed Dent J 1990;14:71-80.

[5] Auguston TE, Morken T. Musculoskeletal problems among dental health personnel: a survey of the public dental health services in Hordaland (in Norwegian). Tidsskr Nor Laegeforen. 96;116:2776-80.

[6] Finsen L, Christensen H, Bakke M. Musculoskeletal disorders among dentists and variation in dental work. Appl Ergon 1998;29(2):119-25.

[7] Chowanadisai S, Kukiattrakoon B, Yapong B, Kedjarune U, Leggat PA. Occupational health problems of dentists in Southern Thailand. Int Dent J 2000;50:36-40.

[8] Szymanska J: Disordes of the musculoskeletal system among dentists from the aspect of Agric Environ Med 2002;9:169-173.

[9] Akesson I, Lundborg G, Horstmann V, Skerfving S: Neuropathy in female dental personnel vibrations. Occup Environ Med 1995;52(2):116-23.

[10] Marshall ED, Duncombe LM, Robinson RQ, Kilbreath SL. Musculoskeletal symptoms in New SouthWales dentists. Aust Dent J 1997;42:240-6.

[11] Milerad E, Ekenvall L. Symptoms of the neck and upper extremities in dentists. Scand J Work

[12] Environ Health 1990;16:129-34.

[13] Shugars D, Miller D, Williams D, Fishburne C, Stricklans D. Musculoskeletal pain among

general dentists. Gen Dent 1987;4:272-6.

[14] Bassett S. Back problems among dentists. J Can Dent Assoc 1983;49:251-6.

[15] Biller FE. Occupational hazards in dental practice.Oral Hygiene 1986;36:1194.

[16] McCrea R. Clinical orthopedic examination. 3rd edition. Churchill Livingston, Longman group, London. UK. 1990.

[17] Ishmael WK, Shorbe HB. Care of the back. 2nd edition. J B Lippincott Comp. Toronto. Canada.1976. Osborn JB, Newwell KJ, Rudney JD, Stollenburg JL. Musculoskeletal pain among Minnesota dental hygienists. J Dental Hyg 1990;64(3):132-138.

[18] Walters E. How to live with failures and stress. Dent manage 1976t 16(11):20- 24.

[19] Fox JG, Jones JM, Occupational stress in dental practice. Br Dent J 1967;123(10):465-473.

[20] Paul E. The technique of seated dentistry. Qunt Int Dent Diag 1979;10:19.

[21] Bers GS. The dentists health. J Am Dent Assoc 1980;100(4):507.

[22] Wille AW. How to avoid occupational hazards of dentistry. Aust Dent J 1967;12(4):348-359.

[23] Green EJ, Brown ME, Body mechanics applied to practice of dentistry. J Am Dent Assoc 1963; 67:679-697.

[24] Visser JL, Straker LM. An investigation of discomfort experienced by dental therapists and assistants at work. Aust Dent J 1994;39(1): 39-44

[25] Stratford PW, Binkley J, Solomon P, Finch E, Gill C, Moreland J. Defining the minimum level of detectable change for the Roland-Morris questionnaire. Phys Ther 1996 Apr; 76(4):359-65; discussion 66-68.

[26] Roland M, Morris R. A study of the natural history of back pain: part I: development of a reliable and sensitive measure of disability in low-back pain. Spine 1983; 8:141-144.

[27] Roland M, Fairbank J. The Roland-Morris Disability Questionnaire and the Oswestry Disability Questionnaire. Spine 2000; 25:3115-3124. 